\title{
DEVELOPMENTS IN ELECTRO-MAGNETIC TOMOGRAPHY INSTRUMENTATION
}

\author{
G M Lyon ${ }^{1}$, Z Z Yu', A J Peyton ${ }^{1} \&$ M S Beck ${ }^{2}$
}

Abstract: A new EMT sensor and instrumentation is described which combines the best features of previous systems and has a modular structure to allow for future system expansion and development.

\section{System Overview}

This paper describes a new electromagnetic tomography[1] instrument which has been developed at UMIST and subsequently at Lancaster University. The new system attempts to combine the best features from two existing systems[2]:

1: The INESC Multi-pole EMT scanner which uses point source solenoid excitation.

2. The UMIST parallel field EMT system which uses a uniform parallel excitation field.

Both these forms of excitation field generation have their own merits; a point source field providing higher measurement independence, in particular near boundary, and hence image resolution. The parallel excitation field provides optimum central object measurement sensitivity[3]. The new sensor and system instrumentation has been designed to operate with either or both of these excitation methods to provide an optimised measurement data set. To realise this objective the new sensor and instrumentation, in its present configuration, uses two sets of 16 independent excitation and detection channels. The following sections describe the sensor and instrumentation designed for the new system.

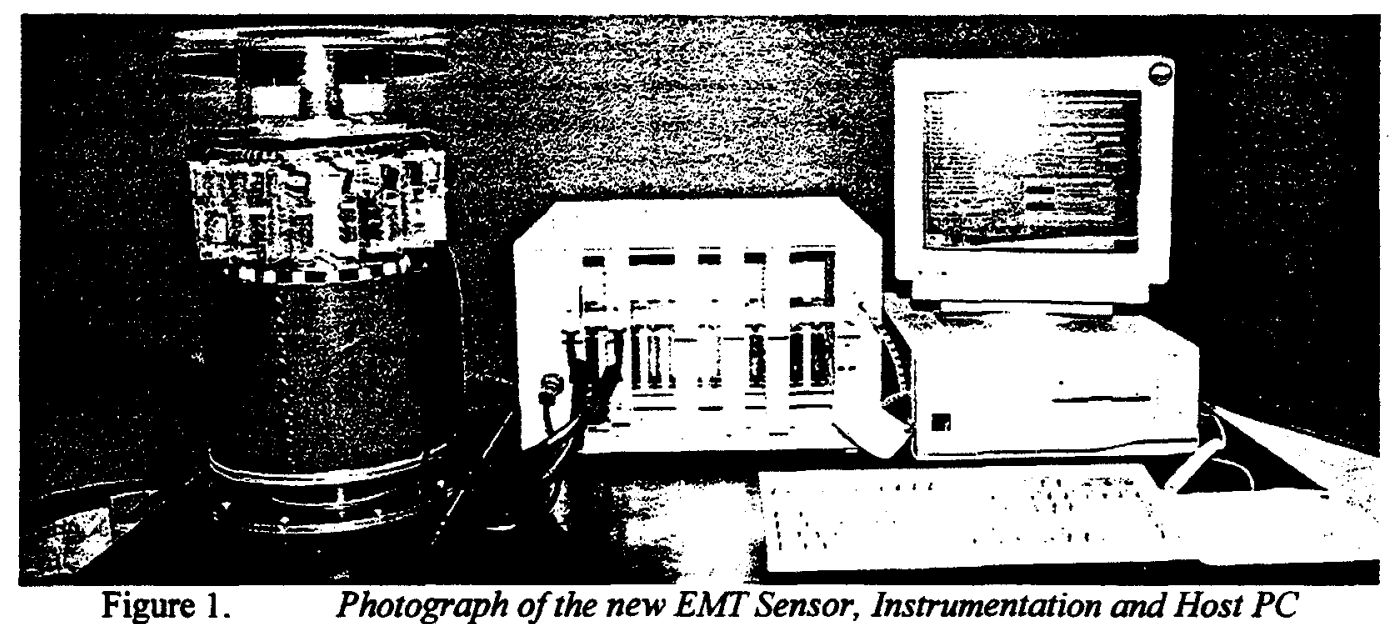

As with the previous EMT systems, AC magnetic field excitation and detection is performed by a set of inductive coils positioned around the boundary of the object space. The excitation coils are driven by an AC sinusoidal electric current and the field generated induces a set of measurable voltages across the detection coils. The sensor is capable of detecting the presence of both magnetic and electrically conductive materials. In general magnetic material increases the mutual inductance between excitation and detection coil(s), resulting in an increased measurement. Conductive materials generally attenuate the measurement due to eddy current losses in the material itself.

\footnotetext{
${ }^{1}$ Engineering Department, Lancaster University, Bailrigg, Lancaster, LA1 4YW.

${ }^{2}$ Process Tomography Unit, UMIST, PO Box 88, Manchester M60 1QD
} 


\section{Sensor Design}

The new sensor array is shown in Figure 2. An 8" diameter object space is surrounded by a set of detection coils, then a layer of excitation coils and finally the outer field confinement screen. This screen is made from ferrite powder and encapsulated between two plastic pipe sections. A metal outer cover then encloses the entire sensor, including a set of small circuit boards used for coil driver and measurement buffer circuitry.

The angular positions of the detection and excitation coils are interleaved to reduce the direct coil-pair coupling. Careful design of the coil size, shape and positioning avoids excessively large background signals and reduces the dynamic range of the sensor signals. These would be 5 times greater if the excitation and detection coils were positioned at the same angle. Theoretically, for certain detection coil geometry's and locations, there are specific null points that can passively cancel the entire background or empty space field signal. In practice, a large reduction in the background signal level is beneficial; reducing the complexity of the signal conditioning electronics.

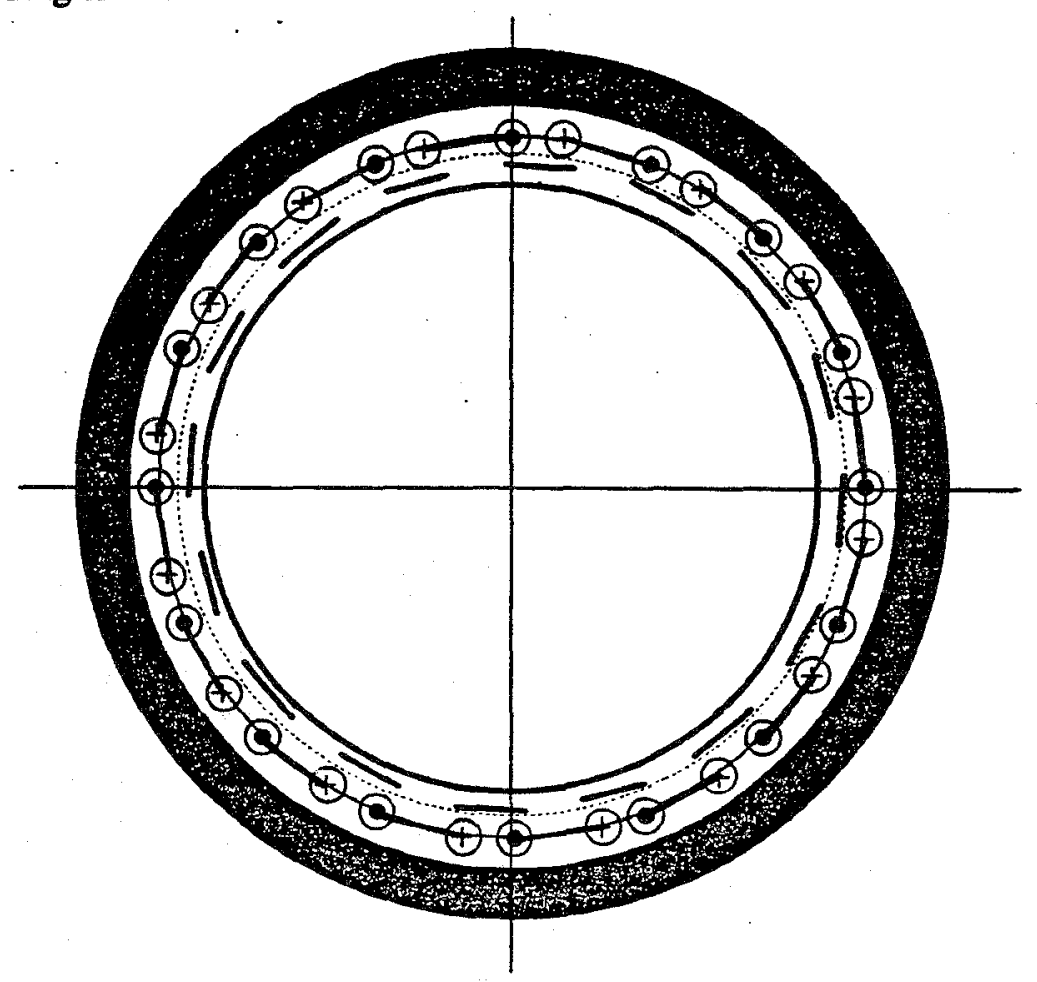

Figure 2 EMT sensor array: cross sectional view

The excitation field can be set by one or more of the excitation coils to control the shape and direction of the energising field. Energising a single coil, in turn, provides 16 point source field patterns. Energising all the excitation coils simultaneously with drive currents which follow a sinusoidal distribution around the sensor produces a near-parallel excitation field. Applying both of these forms of excitation will provide a data set which combines measurements with high spatial independence and measurements with high central object detectability.

\section{System Electronics}

The system electronics, with the exception of a small section of front-end electronics in the sensor itself, has been housed in a VME style equipment case and consists of a number of compatible function modules. All the modules share a common mixed signal backplane which is used for system control and analogue signal distribution. The following subsections describe briefly each module. 
This module generates a set of quadrature sinusoidal reference signals $\{\sin (\omega t), \sin (\omega t+\pi / 2), \sin (\omega t+\pi)$, $\sin (\omega t+3 \pi / 2)\}$. A pair of direct digital synthesis IC's, programmed with identical frequency data are used to drive a pair of high speed digital to analogue converters. A combination of programmable quadrature phase offset and inverting / non-inverting buffers output the desired waveforms. The module has a theoretical (Nyquist limit) upper frequency of $16 \mathrm{MHz}$. In practice an imposed design limit of $2 \mathrm{MHz}$ has been chosen. This allows for good low pass filtering and de-glitching of the DAC outputs in order to provide a smooth sinusoidal output signal. The lower frequency limit is effectively DC and the frequency resolution is less than $0.01 \mathrm{~Hz}$.

\section{Excitation control module}

This 16 channel module generates the drive signals for a set of excitation coils. Independent control of each output is achieved by using a 4-quadrant analogue multiplier. This device multiplies the sinusoidal reference signal with a bipolar DC control voltage, generated by a DAC. Thus, the output signal can be written as:

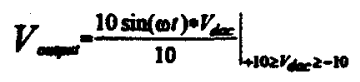

This provides amplitude scaling and phase inversion for each output signal. Also, each channel can be disabled by an analogue switch to allow fast source switching of preset amplitude values.

\section{Programmable gain and compensation module}

This module is used to provide signal conditioning for 16 measurement channels. Each channel has a twinstage programmable gain amplifier with resistor selectable gain settings. In between the two gain stages an external signal can be subtracted from the measurement signal. This feature has been added to provide active compensation; the removal of a synthesised reference (background or empty space) signal component from the measurement signal, thus making more efficient use of the subsequent demodulation and conversion circuitry. The reference signal is synthesised by a second identical excitation control module.

\section{Demodulation module}

This module provides demodulation for a set of 16 input signals. Parallel muitiplying demodulation is performed with respect to both $\sin (\omega t)$ and $\sin (\omega t+\pi / 2)$ producing in-phase and quadrature components. The demodulator's outputs, via suitable low pass filtering, can be written as:

$$
\begin{aligned}
& \bar{V}_{\text {In-Phase }}(\text { volts })=\frac{V_{\text {lmpau }} * V_{\text {Real }- \text { Ref }}}{10}=\left.\frac{K \sin (\omega t+\phi) * 10 \sin (\omega t)}{10} \equiv \frac{K \cos (\phi)}{2}\right|_{\text {LPF } \omega_{c}<<\omega} \\
& \bar{V}_{\text {Quadranure }}(\text { volts })=\frac{V_{\text {lnpou }} * V_{\text {Imag-Ref }}}{10}=\left.\frac{K \sin (\omega t+\phi) * 10 \sin (\omega t+\pi / 2)}{10} \equiv \frac{K \sin (\phi)}{2}\right|_{L P F: \omega_{c}<\infty}
\end{aligned}
$$

Data conversion module

This module uses a semi-parallel structure to achieve a sub $100 \mu \mathrm{S}$ conversion time for 32 demodulated, inphase and quadrature, component signal inputs. Eight 4-channel ADC's are used which incorporate input multiplexing and a conversion data storage FIFO. The ADC configuration registers can be programmed in parallel to enable the module to free-run through a pre-determined sampling sequence when required.

Embedded control and communications module

The final system module, currently under development, will provide embedded control and serial communication facilities. The module is based on the PC104 embedded PC format and has two PC104 sites, to be occupied by a processor and an Ethernet card respectively. This will allow the embedded PC to 
control the entire data collection operation and transfer measurement data to a remote host, perhaps a considerable distance from the instrument itself.

When combined, the modules described above provide the necessary signal generation and processing required for tomographic data collection. Figure 3 shows the signal flow path for a single excitation and a single detection channel. The figure shows how the compensation signal is subtracted from the measurement signal in between the two programmable gain stages, prior to demodulation and conversion. At present the system operates with 16 excitation and 16 detection channels in parallel. In future additional channels, in particular for detection, maybe added.

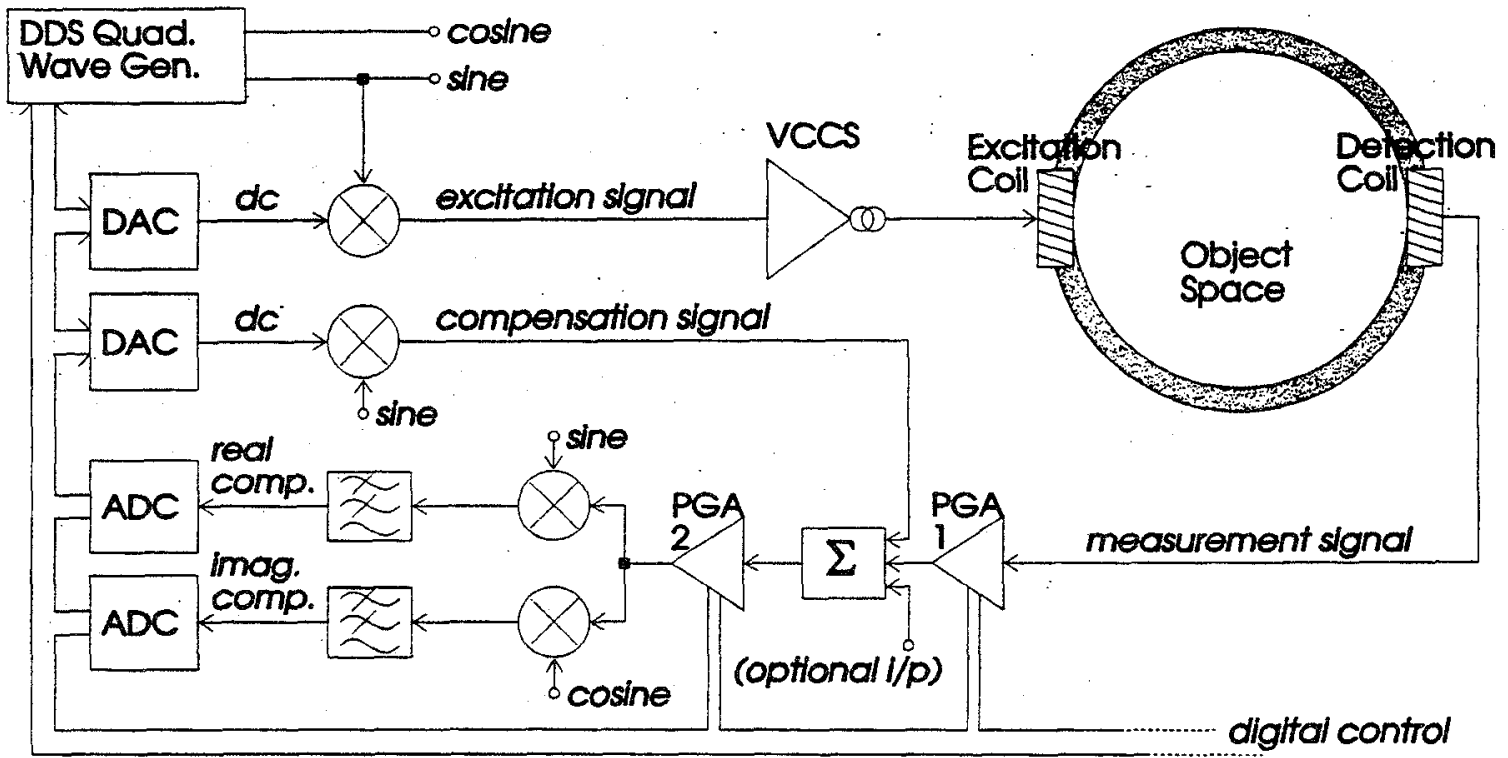

Figure 3. Signal flow path for one excitation and one detection channel

\section{Discussion}

The system presented in this paper can be viewed as a natural progression from the two previous EMT instruments developed at INESC and at UMIST. The new system improves on both the size of the object space and the operational frequency range; up from $100-200 \mathrm{kHz}$ to $2 \mathrm{MHz}$. Future developments will see the integration of this hardware with complimentary image reconstruction software which is being developed by colleagues at INESC-Aviero. This work forms the greater part of a Brite-EuRam funded research project aimed at developing EMT for industrial applications.

\section{References}

[1] Yu et-al 1993 Imaging system based on electromagnetic tomography (EMT). Electron Lett. 29 625-6

[2] Peyton et-al 1996 An overview of electromagnetic inductance tomography: description of three systems. Meas. Sci. Technol. $7261-71$.

[3] Yu et-al 1995 Optimum excitation field for non-invasive electrical and magnetic tomography sensors. Conf. ECAPT (Bergen, Norway) ISBN 0952316528 pp311-20

\section{Acknowledgement}

The work was supported by the CEU Brite EuRam programme (contract BRE2-CT94-0604). 